\title{
Reply to "Displaced distal forearm fractures in children with an indication for reduction under general anesthesia should be percutaneously fixated" by van Egmond PW, Schipper IB, van Luijt PA in Eur J Orthop Surg Traumatol. 2012 Apr;22(3):201-207
}

\author{
Georg Singer · Tanja Peschaut $\cdot$ Tanja Kraus • \\ Robert Eberl
}

Received: 16 April 2012/ Accepted: 30 April 2012/Published online: 15 May 2012

(C) Springer-Verlag 2012

\section{Dear Editor,}

With great interest we read the article "Displaced distal forearm fractures in children with an indication for reduction under general anesthesia should be percutaneously fixated" by van Egmond et al. [1]. We agree with the authors that displaced forearm fractures are commonly encountered injuries in childhood. In our own publication, we have shown that distal forearm fractures account for almost a tenth of all pediatric fractures [2].

The authors show that $43.7 \%$ of the children treated with unfixed reduction of a displaced forearm fracture needed a secondary procedure under general anesthesia. Comparable high rates of re-displacement have been described previously [3]. These high rates of re-dislocations motivated us to perform a retrospective analysis of our own patients. Between 2007 and 2011, we treated 109 children (mean age 8.9, range 2-15 years) with 111 displaced distal forearm fractures with closed reduction and cast immobilization under general anesthesia. Five of these fractures $(4.5 \%)$ were complicated by re-displacement necessitating secondary intervention consisting of closed reduction and cast immobilization in two patients, closed reduction and internal plate fixation in one patient (15 a) and open reduction and internal $\mathrm{K}$-wire fixation in one other child (12 a).

In addition to anatomical reduction and meticulous follow-up, a good cast is the key to prevent re-displacement of conservatively treated fractures. The pivotal role of cast quality has already been addressed by other authors
[4]. Can methodical differences in applying the plaster cast, therefore, explain the abovementioned differences in the percentage of re-dislocations? In our department, the main goal—when applying the cast—is avoiding excessive padding and poor molding. Additionally, our patients were followed on a weekly basis for a mean of 4.6 weeks (range 3-8 weeks) until radiographic union was achieved and the cast could be removed. These facts may explain the low percentage of re-displacements necessitating secondary intervention in our series of patients.

In conclusion, we cannot agree with the authors' recommendation to primary perform invasive fixation of dislocated distal forearm fractures in children.

\section{References}

1. van Egmond PW, Schipper IB, van Luijt PA (2012) Displaced distal forearm fractures in children with an indication for reduction under general anesthesia should be percutaneously fixated. Eur J Orthop Surg Traumatol 22:201-207

2. Schalamon J, Dampf S, Singer G et al (2011) Evaluation of fractures in children and adolescents in a Level I Trauma Center in Austria. J Trauma 71:E19-E25

3. Zamzam MM, Khoshhal KI (2005) Displaced fracture of the distal radius in children: factors responsible for redisplacement after closed reduction. J Bone Jt Surg Br 87:841-843

4. Bhatia M, Housden PH (2006) Re-displacement of paediatric forearm fractures: role of plaster moulding and padding. Injury 37:259-268 